\title{
POSTWEANING HOUSING CONDITIONS AND PARTNER PREFERENCE AND SEXUAL BEHAVIOR OF NEONATALLY ATD-TREATED MALE RATS
}

\author{
Julie Bakker, Jan van Ophemert, and A. Koos Slob \\ Department of Endocrinology and Reproduction, Faculty of Medicine and Health Sciences, \\ Erasmus University, P.O. Box 1738, 3000 DR Rotterdam, The Netherlands
}

(Received 22 November 1993; in final form 26 May 1994)

\begin{abstract}
SUMMARY
Male rats were neonatally treated with cholesterol or a substance that blocks the aromatization of testosterone to estradiol (1,4,6-androstatriene-3,17-dione: ATD). At weaning (21 days) they were either housed alone or in small groups (2-3 animals) and tested for partner preference behavior (PPB) in adulthood. Choice was between an estrous female and an active male (Part I) and between an estrous female and an ATD-male (Part II). Tests were carried out in a 3-compartment box. Social isolation did not have major effects on PPB except when sexual interaction with the stimulus animals was prevented (Part I). In this case, isolates (ATD and control) showed higher preference scores (PS) for the estrous female and spent less time in the empty middle compartment. When the choice was between an estrous female and an ATD-male, partner PS decreased in all males, most clearly in ATD-males. The latter animals spent more time with the stimulus ATD-male than they had done in previous PPB tests with the normal stimulus male. In contrast to partner preference behaviors, sexual behavior was clearly affected by social isolation. Isolates (ATD and control) displayed lower frequencies of mounts and intromissions. These effects persisted over consecutive tests. Ejaculation was not affected. In conclusion, the present results confirm earlier findings about the significance of neonatal endocrine conditions for the organization of adult PPB in male rats. The presence or absence of social conspecifics after weaning appears to have little influence on adult PPB.
\end{abstract}

Keywords-Male rats; Neonatal ATD treatment; Housing conditions; Partner preference behavior; Sexual behavior.

\section{INTRODUCTION}

THE TWO MAJOR factors known to affect adult sexual and related behaviors in male rats are the relative amounts of estradiol present during a critical or sensitive perinatal period (Bakker et al., 1993a; Baum, 1979; Brand et al., 1991; Vega Matuszczyk, 1993; Ward \& Reed, 1985) and social stimulation during prepuberal development (Gerall et al., 1967; Spevak et al., 1973; Ward \& Reed, 1985; Zimbardo, 1958).

Adult gonadally intact male rats treated shortly after birth with ATD (1,4,6-androstatriene-3,17-dione), which inhibits the aromatization of testosterone $(T)$ to estradiol $\left(\mathrm{E}_{2}\right)$ (Brodie et al., 1979; Kaplan \& McGinnis, 1989; Lieberburg et al., 1977), readily display

Address correspondence and reprint requests to: Julie Bakker. 
"masculine" sexual behaviors (i.e., mounts and intromissions) but no or few ejaculations when paired with an estrous female. Such ATD-males also easily show "feminine" sexual behaviors (i.e., proceptive behaviors and lordosis) when paired with an active male (e.g., Bakker et al., 1993b; Brand et al., 1991; Davis et al., 1979; Vreeburg et al., 1977). Thus, these ATD-males are behaviorally less masculinized and less defeminized than normal male rats. Comparable results with ATD were obtained in ferrets (Baum \& Tobet, 1986; Tobet \& Baum, 1987). ATD-males also show a significantly lower partner preference (choice: estrous female vs. active male) for the estrous female than control males (Bakker et al., 1993a, 1993b; Brand et al., 1991). Additionally, partner preference behavior (PPB) and sexual behavior of ATD-males but not of control males, show a nocturnal rhythm. In the late part of the dark phase of the LD cycle the partner preference for the estrous female and frequencies of masculine sexual behaviors, including ejaculations, are significantly higher than in the early part of the dark phase. Feminine sexual behaviors (proceptive and receptive behaviors) also show a nocturnal rhythmicity, with highest frequencies early in the dark phase (Bakker et al., 1993b).

It has been reported that housing conditions can affect the development of adult sexual behavior of the rat (e.g Gerall et al., 1967; Spevak et al., 1973). Male rats singly housed from weaning (age 14 days) were at 90 days of age significantly less sexually active, with no or low intromission frequencies than males housed in groups (Gerall et al., 1967). Male isolates were highly interested in the estrous female, but failed to mount and clasp the posterior region of the female. This effect was persistent over nine consecutive tests. Only living with females for at least 3 weeks somewhat improved the copulatory performance of isolated males in pair tests. Compared with group-housed male rats, these singly housed (from weaning onward) males also showed in adulthood higher levels of aberrent responses, that is, improper orientation towards the female, and hyperexcitable responses such as headshaking, leaping, and tunneling (Gerall et al., 1967). It has been proposed that play activities during the juvenile and puberal phases are essential for the development of normal adult sexual behavior (Gerall et al., 1967; Gruendel \& Arnold, 1969; Spevak et al., 1973). If, because of isolation during development, these play activities are prevented from occurring, they tend to reappear with greater intensity in adulthood when isolates are tested for sexual behavior. Thus, it was hypothesized that when isolated males are confronted with a social stimulus such as an estrous female in a mating test, play responses would be dominant over sexual ones, resulting in inadequate mating (Gerall et al., 1967; Gruendel \& Arnold, 1969; Spevak et al., 1973).

Beach $(1942,1958)$ found no significant differences in adult sexual behavior between animals housed in isolation or in groups from weaning either at the age of 14 days or at 21 days. Kagan and Beach (1953) even reported an enhancing effect of social isolation on adult sexual behaviors (increase in intromission and ejaculation frequencies, shorter latencies to onset of copulation). However, the design and conclusions of this latter study were criticized by Larsson (1956). Other studies investigating the effects of different pre- and postweaning housing conditions on sexual behavior have given rather consistent results, that is, decrease in frequencies of sexual behaviors (Gerall et al., 1967; Gruendel \& Arnold, 1969; Spevak et al., 1973; Zimbardo, 1958). Most studies were undertaken to investigate the effects of housing conditions on sexual performance only, and not on motivational aspects of sexual behavior such as partner preference behavior. Only Zimbardo (1958) investigated a possible interaction between the effects of shocking a young male rat for approaching a receptive female (early avoidance trairing) and housing condi- 
tions. He did not find any interaction between early avoidance training and housing conditions.

Partner preference can be defined as the preference of an animal to spend more time and/ or to interact sexually more with one animal than with another when given the choice between two stimulus animals (e.g., an estrous female and a sexually active male) (Adkins-Regan, 1988; Bakker et al., 1993a; Slob et al., 1987). Adult ATD-males housed together readily mount each other and display lordosis behavior in response; they also show various proceptive behaviors (hop \& dart, presenting, earwiggling). Especially the frequent display of feminine sexual behaviors of these ATD-males is quite different from what can be observed in cages with normal intact male rats. Therefore, it could be hypothesized that sexual experiences of socially housed ATD-males could affect their partner preference behavior differently from singly housed ATD-males and control males.

This hypothesis, that is, the possible interaction between neonatal hormone treatment and housing conditions from weaning onward, was investigated in the present study (Part I). Adult partner preference behavior and sexual behaviors were studied in neonatally ATD-treated males and control males, caged either alone (isolates) or in small groups (2-3 animals).

When no effect of housing conditions was found, we changed the stimulus animals in the partner preference tests (Part II): an ATD-male (instead of a "normal" male), and an estrous female. This was done to investigate whether ATD-males housed in groups would prefer, compared to ATD isolates, the stimulus ATD-male over a normal stimulus male.

\section{METHODS}

\section{Animals}

Experimental animals were Wistar albino rats obtained from a commercial breeder (Harlan Sprague-Dawley, Zeist, The Netherlands). The male stimulus animals were F1 hybrids of two inbred Wistar strains $(R \times U)$, the female stimulus animals were Wistar albino rats. Food and water were available ad lib. The animals were kept in a 14:10 h light:dark cycle (lights on from 1745 to $0745 \mathrm{~h}$ ). The temperature in the animal room ranged from 20 to $22^{\circ} \mathrm{C}$.

\section{Treatments}

Female rats were time-mated. Parturition occurred 22 days later (day of birth = Day 0 ). Within 2-4 h after birth, the newborn males received subcutaneously (SC) a silastic capsule (SR3: inner diameter $1.5 \mathrm{~mm}$, outer diameter $2.1 \mathrm{~mm}$, length $5 \mathrm{~mm}$ ) containing ATD or cholesterol. The implants were removed at weaning, 21 days of age. In the present experiments 21 ATD-males (out of 10 litters) and 9 control males (out of 4 litters) were used.

At weaning, the animals were randomly divided into two groups: 4 control males and 10 ATD-males were housed singly; 5 control males and 11 ATD-males were caged in groups, i.e. 2-3 rats to a cage of the same treatment. The males were left undisturbed until the onset of behavioral testing at the age of approximately 3 mo.

Stimulus animals, tested repeatedly, were sexually active males and estrous females. The stimulus females were ovariectomized and brought into behavioral estrous by injecting $20 \mu \mathrm{g}$ estradiol benzoate (EB), $24-48 \mathrm{~h}$ prior to testing, followed by $1.0 \mathrm{mg}$ progesterone (P) 3-4 h before testing. These hormones were dissolved in olive oil and injected $\mathrm{SC}$ in the neck. 


\section{Behavioral Testing}

Three-compartment partner preference test. A test box of gray Perspex with a transparent front was used. It was divided in three compartments $(60 \times 30 \times 40 \mathrm{~cm}$ each) with a small opening $(13 \times 12 \mathrm{~cm})$ in both partitions near the front window (Slob et al., 1987). These openings could be closed by a sliding door. Stimulus animals were either tethered with a rope to the rear of one of the lateral compartments or could freely move behind a wire mesh separation halfway down the lateral compartment. The tethered animals thus had a limited action radius. When physical interaction was prevented by wire mesh, the experimental animal could only see, smell, or hear the stimulus animals.

Before testing, two stimulus animals and the experimental animal were put in the test apparatus, one in each compartment, with the sliding doors closed, for $15-20 \mathrm{~min}$ adaptation. At the beginning of the test (which lasted $15 \mathrm{~min}$ ), the sliding doors were removed and the experimental animal could freely move around and interact with the stimulus animals or stay before the wire mesh separation. The time spent in each compartment was recorded. Various sexual behaviors with the tethered stimulus animals were scored. Masculine sexual behavior was measured by the number of mounts plus intromissions and ejaculation frequencies.

To quantify partner preference behavior, a preference score was calculated for each test by subtracting the time spent in the compartment with the sexually active male (or the ATD-male in Part II) from the time spent in the compartment with the estrous female (Edwards \& Pfeifle, 1983). Thus, a positive score indicates a preference for the estrous female; a negative score indicates a preference for the sexually active male (Slob et al., 1987).

Pair-test with sexually active male. After $5 \mathrm{~min}$ of adaptation of the stimulus male, an experimental male was introduced in a semicircular cage. The lordosis responses of the experimental male to the mounting of the stimulus male were recorded. The test lasted until the experimental animal had received 10 mounts or 10 min had elapsed. In the data analyses, the lordosis quotients of all males who received 4 or more mounts by the stimulus male are included.

\section{Test Procedure}

Behavioral tests started when the males were approximately 3 mo old. Firstly, four tests were performed twice weekly without sexual interaction possible (stimulus animals behind wire mesh separation; Tests 1-4). Then six tests were carried out twice weekly with sexual interaction possible (tethered stimulus animals; Tests 5-10). All tests were performed in the early part of the dark phase (between $0830-1100 \mathrm{~h}$ ) of the LD cycle except Test 9, which was carried out in the late part of the dark phase (between 1400-1630h). Following the last partner preference test (Test 10), the males were tested for feminine sexual behavior, that is, lordosis in a pair-test with a sexually active male.

Part II started 1 week after Test 10. Males were subjected to three weekly partner preference tests with an estrous female and a gonadally intact sexually experienced ATDmale as stimulus animals (Tests 11-13). In Test 12, the females received a vaginal mask to prevent intromissions.

\section{Statistics}

The behavioral data were subjected to MANOVA (multivariate analysis of variance), followed by the least significant difference (LSD)-procedure (Kirk, 1968). Only significant $F$ values are presented. The lordosis data were analyzed using the Fisher's exact one- 
tailed probability test (number of animals showing lordosis behavior) and using the Student's $t$-test (the lordosis quotients). The $p<.05$ level was used as the upper limit for statistical significance.

\section{RESULTS}

Part I: Partner Preference Behavior With an Estrous Female and an Active Male as Stimulus Animals

Partner preference behavior. Partner preference behavior data are shown in Fig. 1. MANOVA on the preference scores without sexual interaction possible (stimulus animals behind wire mesh; see Fig. 1A) showed a significant effect of housing conditions, $F(1$, 26) $=4.52, p=.043$, and of testing, $F(3,78)=3.33, p=.024$. Overall, male rats housed in isolation showed a significantly higher partner preference for the estrous female than male rats housed socially. The preference for the female partner increased significantly in all males over the tests. In the fourth test, isolates (ATD and controls) showed a low preference for the estrous female (mean $338 \pm 33 \mathrm{~s}$ ), whereas socially housed males (ATD and controls) showed no clear partner preference (mean $146 \pm 83 \mathrm{~s}$ ). There were no significant differences between ATD and control males in partner preference behavior without sexual interaction possible. MANOVA on the time spent in the empty middle compartment (see Fig. 1B) showed a significant effect of housing conditions, $F(1,26)=$ $5.11, p=.032$, and a significant group effect, $F(1,26)=4.35, p=.047$.

Overall, isolates (ATD and controls) spent significantly less time in the empty middle compartment than socially housed males (ATD and controls). Group comparisons revealed that control males spent significantly less time in the empty middle compartment than ATD-males. In Fig. 1C the time spent with the estrous female and the time spent with the active male is shown. From looking at this figure, it is clear that isolates (ATD and controls) spent more time with the stimulus animals than males socially housed (ATD and controls).

MANOVA on the partner preference data with sexual interaction possible (stimulus animals tethered; Fig. 1D) showed a significant effect of groups, $F(1,26)=54.48, p<$ .001 , and a significant Group by Test interaction, $F(5,130)=2.46, p=.036$. Further analysis with $\operatorname{LSD}(5 \%=240 \mathrm{~s})$ showed that the preference for the female partner increased significantly in all males, with ATD-males showing a significantly lower preference for the estrous female partner than control males. There were no differences in partner preference behavior with sexual interaction between isolated males and socially housed males. When the males were tested in the late part of the dark phase (Test 9), the preference for the female partner increased almost significantly in ATD-males $[\operatorname{LSD}(5 \%)=240 \mathrm{~s}]$.

MANOVA on the time spent in the empty middle compartment (Fig. 1E) revealed an effect of groups, $F(1,26)=24.04, p<.001$, and a significant Group by Test interaction, $F(5,130)=5.10, p<.001$. Overall, ATD-males spent significantly more time in the empty middle compartment than control males. Further analysis with LSD $(5 \%=120$ s) showed that the time spent in the middle compartment significantly decreased over tests in control males. The time spent with the estrous female and the time spent with the active male is shown in Fig. IF. From looking at this picture, it is clear that ATDmales spent more time with the active male and less time with the estrous female than control males. No clearcut differences between singly and socially housed males, ATD 

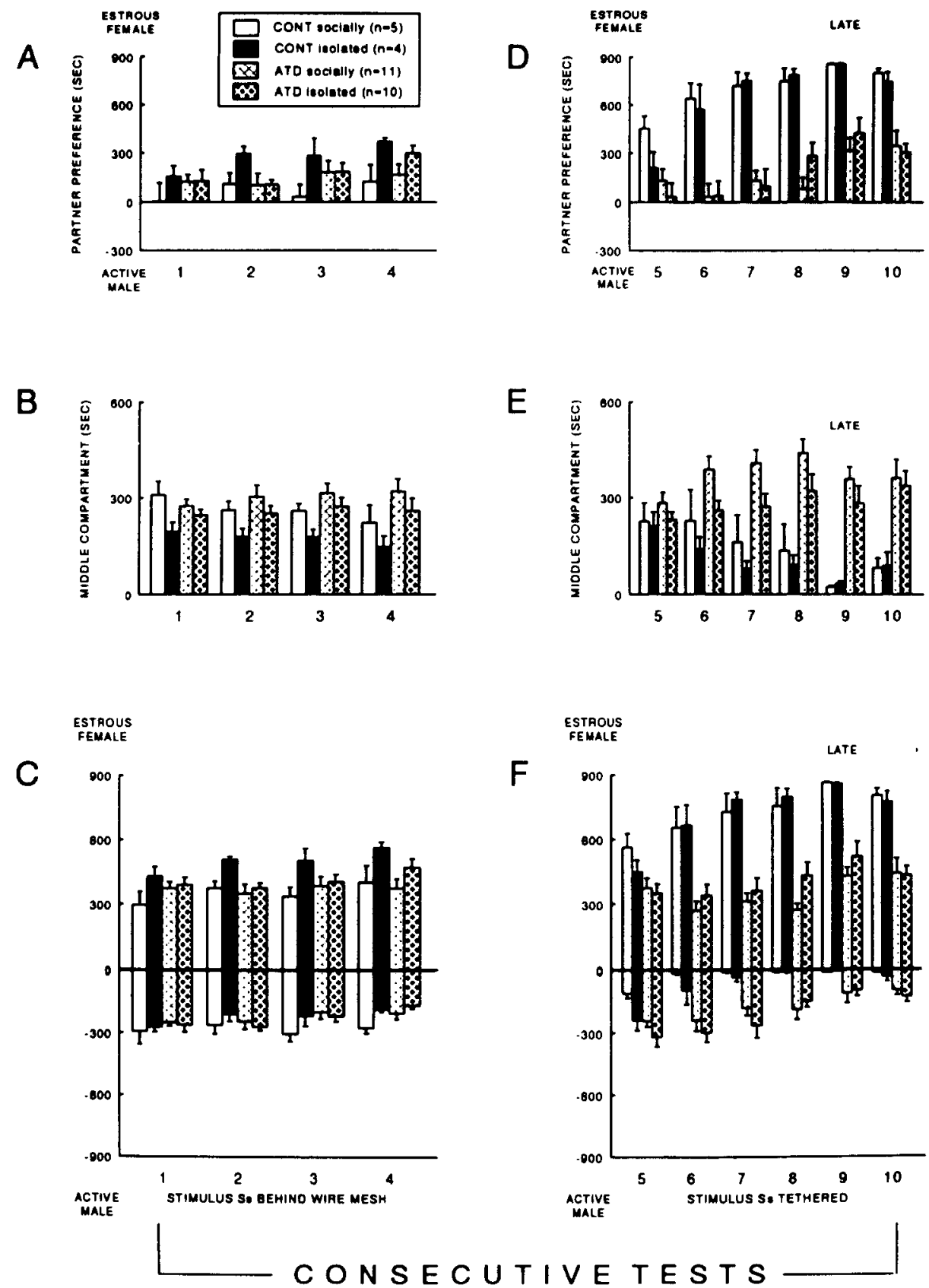

FIG. 1: Partner preference behavior in a three-compartment box (3CB) of adult, gonadally intact male rats, neonatally treated with the aromatization blocker ATD or cholesterol (controls). Male rats (ATD and control) were either housed alone (isolated) or housed in small groups (socially). In Tests 1-4 (A-C) the stimulus animals (an estrous female and an active male) were behind a wire mesh separation; in Tests 5-10 (D-F) the stimulus animals were tethered. Tests 1-8 and 10 were carried out in the early part of the dark phase; Test 9 in the late part of the dark phase. (A) shows partner preference behavior; (B) shows the time spent in the empty middle compartment; and (C) shows the time spent with the estrous female and the time spent with the active male. (D) shows partner preference behavior; (E) shows the time spent in the empty middle compartment; and (F) shows the time spent with the estrous female and the time spent with the active male. Values are means and SEM. 

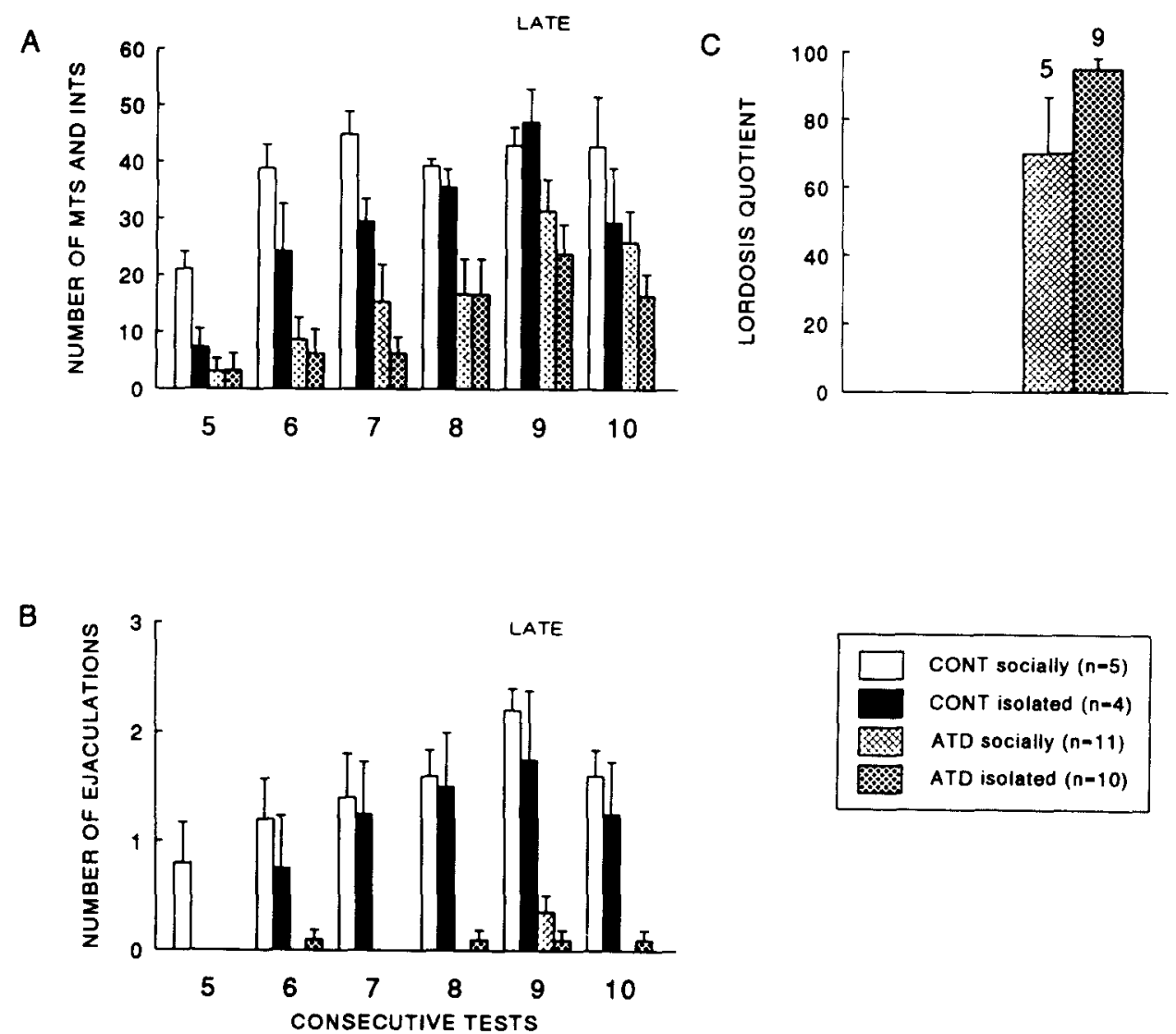

FIG. 2: Sexual behaviors of isolated and socially housed ATD and control male rats with the tethered stimulus female during partner preference testing $(A, B)$ and lordosis behavior in a pairtest with an active male $(C)$. Tests 5-8 and 10 were performed in the early part of the dark phase; Test 9 in the late part of the dark phase. (A) shows the total number of mounts plus intromissions with the estrous female. (B) shows the number of ejaculations. (C) shows the lordosis quotients of adult male rats and the number of ATD male rats mounted by the active male. Values are means and SEM.

as well as controls, can be seen. In the course of testing, the preference for the estrous female became more and more obvious.

Sexual behavior with the tethered estrous female during partner preference testing. The mean number of mounts plus intromissions are depicted in Fig. 2A. MANOVA indicated a significant effect of housing conditions, $F(1,26)=7.06, p=.013$, a significant group effect, $F(1,26)=51.57, p<.001$, a significant effect of testing, $F(5,130)=32.29$, $p<.001$, and a significant Group by Test interaction, $F(5,130)=3.11, p=.011$. Isolates (ATD and control) displayed significantly fewer mounts and intromissions than socially housed males (ATD and control). Furthermore, ATD-males showed lower frequencies of masculine sexual behaviors (mounts and intromissions) than control males. Overall, the number of mounts and intromissions increased significantly in all males over the tests.

Ejaculation frequencies are shown in Fig. 2B. ATD-males almost never ejaculated 
when tested early in the dark phase. Late in the dark phase (Test 9) social ATD-males started to ejaculate ( 4 of 11 vs. 0 of 11 in all "early" tests). ANOVA on the ejaculation frequencies of control males revealed only a significant effect of tests, $F(5,35)=6.76$, $p<.001$. The number of ejaculations increased in both control groups between Tests 5 and 10. None of the control isolates ejaculated in the first test (Test 5), whereas 3 of 5 socially housed controls ejaculated. In the second test (Test 6) 2 of 4 isolates ejaculated vs. 4 of 5 social males. In the third test the differences had disappeared: 3 of 4 isolates vs. 4 of 5 social control males. By the end of the partner preference testing (Test 10) all control males ejaculated, with the exception of one singly housed control male.

Sexual pair test with active male. The lordosis data are shown in Fig. 2C. Control males did not display any lordosis behavior. Significantly more singly housed than socially housed ATD-males were mounted by the stimulus male: 9 of 11 isolates vs. 5 of 11 socials (Fisher's exact one-tailed probability test $p=.04$ ). The lordosis quotients did not differ significantly between the two ATD-groups.

\section{Part II: Partner Preference Behavior With an Estrous Female and an ATD-Male as Stimulus Animals}

Partner preference behavior. The preference scores are depicted in Fig. 3. For reasons of comparison the results of Test 10 (last test with choice between estrous female and active male) are also shown and included in the statistical analysis. MANOVA on the data of Tests 10-13 (Fig. 3A) revealed a significant effect of groups, $F(1,26)=48.08$, $p<.001$, and a significant effect of testing, $F(3,78)=4.28, p=.008$. The preference for the estrous female decreased significantly in all males in Tests 11-13 with an ATDmale as stimulus male compared to Test 10 with an active male as stimulus male.

MANOVA on the middle compartment data (Fig. 3B) showed a significant effect of groups, $F(1,26)=14.3, p=.001$, and a significant effect of tests, $F(3,78)=3.56, p=$ .018. The time spent in the empty middle compartment increased in all males over tests. In Fig. 3C, the time spent with the estrous female and the time spent with the stimulus male is depicted. From this figure, it is clear that both ATD-male groups spent more time with the stimulus ATD-male than with the active male.

Sexual behavior with the tethered estrous female during partner preference testing. MANOVA on the total number of mounis and intromissions (Fig. 3D) revealed a significant effect of housing conditions, $F(1,26)=7.26, p=.012$, a significant group effect, $F(1,26)=35.92, p<.001$, a significant effect of tests, $F(3,78)=10.49, p<.001$, and a significant Group by Test interaction, $F(3,78)=2.79, p=.046$. Overall, isolates (ATD and control) displayed fewer mounts and intromissions than socials (ATD and control). Subsequent LSD analysis $(5 \%=9.5)$ of the group by test interaction showed that the number of mounts and intromissions in control males fluctuated over tests, with the highest frequencies in Test 12 when intromissions were prevented by a vaginal mask of the stimulus female. The mount and intromission frequencies did not change in ATDmales over tests.

Ejaculation frequencies are shown in Fig. 3E. ANOVA on the data of control males in Tests 10,11 , and 13 (Test 12 is excluded from statistical analysis because intromissions and ejaculations were prevented by vaginal occlusion of stimulus females) showed a significant effect of testing, $F(2,14)=17.39, p<.001$. The ejaculation frequencies increased significantly between Tests 10 and 13 in control males. 

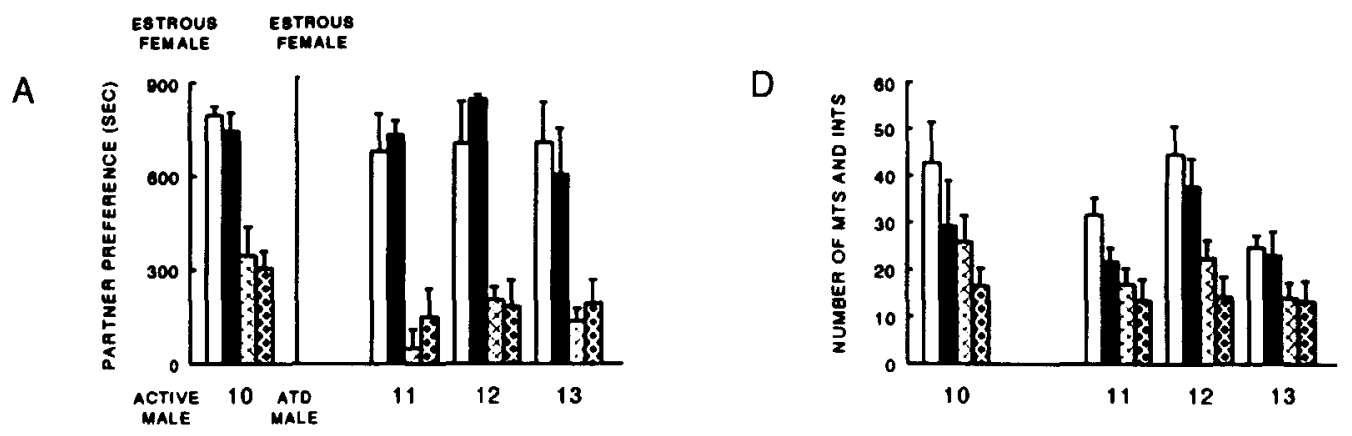

B

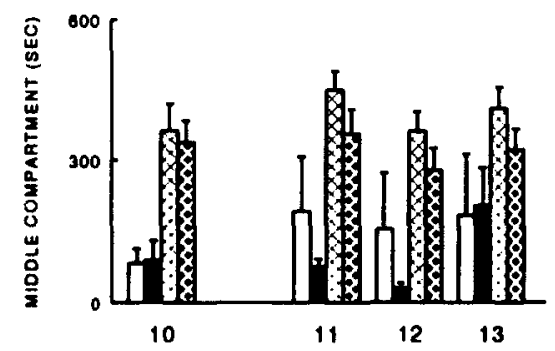

ESTROUS ESTROUS

FEMALE FEMALE

C

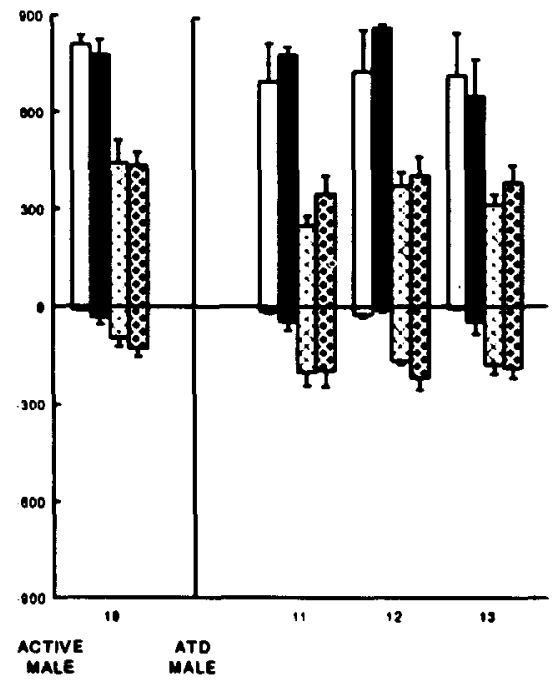

$E$

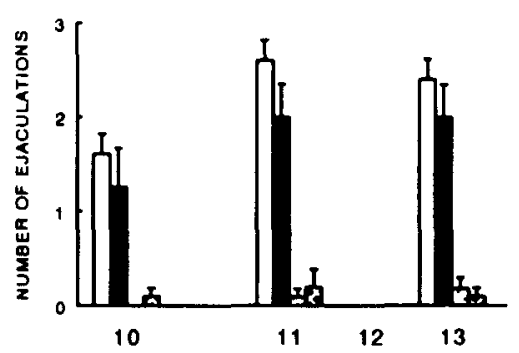

CONT socially $(n-5)$

CONT isolated $(n=4)$

ATD socially $(n-11)$

ATD isolated $(n-10)$

FIG. 3: Partner preference behavior in the three-compartment box of adult, gonadally intact male rats neonatally treated with the aromatization blocker ATD or cholesterol (controls). Male rats were either housed alone (isolated) or housed in small groups (socially). For reasons of comparison the results of Test 10 (see Fig. 1; last test with choice between estrous female and active male) are also shown. In Test 12 intromissions were prevented by vaginal occlusion of the stimulus females. (A) shows partner preference behavior; (B) shows the time spent in the empty middle compartment; and (C) shows the time spent with the estrous female and the time spent with the ATD-male. (D) shows the total number of mounts plus intromissions with the estrous female during partner preference testing. (E) shows the number of ejaculations. Values are means and SEM. 


\section{DISCUSSION}

From the present experiments, it is clear that postweaning social conditions did not have major effects on adult partner preference behavior of gonadally intact ATD and control male rats. Housing conditions only affected partner preference behavior when no social interaction with the stimulus animals was possible. Then, the isolated male rats (ATD and control) showed a higher partner preference for the estrous female and spent less time in the empty middle compartment. When social interaction with the stimulus animals was possible, no differences were found between isolated and socially housed male rats (ATD and control).

Partner preference behavior was clearly affected by neonatal treatment with ATD, which corroborates earlier results from our laboratory (Bakker et al., 1993a, 1993b; Brand et al., 1991). When the animals were offered a choice between an estrous female and an ATD-male (instead of a "normal" male) partner, preference scores decreased in all males, but most clearly in the experimental ATD-males. The latter males spent less time with the estrous female, and more time with the stimulus ATD-male. The hypothesis suggested in the Introduction could not be supported: social ATD-males compared with ATD isolates, did not show a higher preference for the stimulus ATD-male. Furthermore, ATD-males spent more time with the stimulus ATD-male than in previous PPB tests with the normal stimulus male (estrous female vs. normal male).

The stimulus ATD-males were regularly mounted by the experimental males (both ATD and control males) and showed high frequencies of proceptive and receptive behaviors. The stimulus ATD-males did not mount the experimental males. Presumably, being tethered rendered the stimulus ATD-males more likely to display their "feminine" behaviors than to show their "masculine" behaviors. Thus, the experimental male could mount and intromit the estrous female or mount the stimulus ATD-male. The clear preference for the estrous female over the stimulus ATD-male displayed by control males, could be explained by the fact that with the estrous female intromissions and ejaculations were possible, whereas the stimulus ATD-male could only be mounted. However, preventing intromissions and ejaculations by vaginal occlusion of the estrous female (albeit in only one test), did not change the partner preference behavior of the control males. Maybe, other factors than penile stimulation by intromission play an important role in determining the preference for the estrous female in control males. Further research should address this possibility.

Although partner preference behavior was not changed by postweaning social conditions, sexual behaviors during partner preference testing clearly were affected. Isolated males displayed fewer mounts and intromissions than socially housed males. These effects were persistent over tests. Ejaculatory behaviors were not affected.

The question why social isolation causes a permanent detrimental effect on adult sexual behavior in male rats needs to be addressed. It was postulated that a lack of play behavior in the early isolated males makes them more "playful" and less "sexual" when tested in adulthood with an estrous female rat (see also Introduction; Gerall et al., 1967). In the present study we did not observe that the isolated males showed more play behaviors during partner preference behavior testing than the socially housed males. Others postulated that the "deprivation of opportunities of practicing climbing during infancy" (Hård \& Larsson, 1968) resulted in retarded sexual development. Once a normal mount was performed, a normal mating pattern followed (Hård \& Larsson, 1968). The latter authors showed that most isolated males "overcame" their isolation 
effect and became normal copulators, whereas a minority of their isolated males never started to copulate. In the present study, we did not find such a phenomenon: all isolated control and ATD-male rats (with one exception) displayed mounting and intromission behaviors, albeit with lower frequencies than the socially housed males.

Finally, a new finding emerged from the present investigation. Although the lordosis quotients of ATD-male rats did not differ between isolates and socials, the isolated ATDmale rats seemed to be more sexually attractive. When pair-tested with a normal stimulus male, significantly more isolated ATD-males ( 9 of 10 ) were mounted than socially housed ATD-males (5 of 11). We have no explanation for this finding and future research is needed.

As was hypothetized in the introduction, we expected an effect of housing conditions on partner preference behavior. Especially in ATD-male rats, we assumed that the frequent occurrence of mounting and lordosis in the homecage could affect their partner preference behavior, that is, induce a more male-oriented preference. The present results do not support this idea.

In conclusion, the present results emphasize the importance of neonatal endocrine conditions for the organization of adult partner preference behavior in the male rat. Apparently, the presence or absence of social conspecifics from weaning onward plays no role in determining adult partner preference behavior. Future research with the ATDmale exposed to various choices of sex partners are needed to shed more light on the interesting phenomenon of sexual partner preference behavior.

\section{REFERENCES}

Adkins-Regan E (1988) Sex hormones and sexual orientation in animals. Psychobiology 16:335-347.

Bakker J, Brand T, van Ophemert J, Slob AK (1993a) Hormonal regulation of adult partner preference behavior in neonatally ATD-treated male rats. Behav Neurosci 107:480-487.

Bakker J, van Ophemert J, Slob AK (1993b) Organization of partner preference and sexual behavior and its nocturnal rhythmicity in male rats. Behav Neurosci 107:1049-1058.

Baum MJ (1979) Differentiation of coital behavior in mammals: A comparative analysis. Neurosci Biobehav Rev 3:265-284.

Baum MJ, Tobet SA (1986) Effect of prenatal exposure to aromatase inhibitor, testosterone, or antiandrogen on the development of feminine sexual behavior in ferrets of both sexes. Physiol Behav 37:111-118.

Beach FA (1942) A comparison of copulatory behavior of male rats raised in isolation, cohabitation and segregation. J Gen Psychol 60:121-136.

Beach FA (1958) Normal sexual behavior in male rats isolated at fourteen days of age. J Comp Physiol Psychol 51:37-38.

Brand T, Kroonen J, Mos J, Slob AK (1991) Adult partner preference and sexual behavior of male rats affected by perinatal endocrine manipulations. Horm Behav 25:323-341.

Brodie AMH, Marsh DA, Wu JT, Brodie HJ (1979) Aromatase inhibitors and their use in controlling oestrogen-dependent processes. J Steroid Biochem 11:107-112.

Davis PG, Chaptal CV, McEwen, BS (1979) Independence of the differentiation of masculine and feminine sexual behavior in rats. Horm Behav 12:12-19.

Edwards DA, Pfeifle, JK (1983) Hormonal control of receptivity, proceptivity and sexual motivation. Physiol Behav 30:437-443.

Gerall HD, Ward IL, Gerall AA (1967) Disruption of the male rat's sexual behavior induced by social isolation. Anim Behav 15:54-58.

Gruendel AD, Arnold WJ (1969) Effects of early social deprivation on reproductive behavior of male rats. J Comp Physiol Psychol 67:123-128.

Hård E, Larsson K (1968) Dependence of adult mating behavior in male rats on the presence of littermates in infancy. Brain Behav Evol 1:405-419. 
Kagan J, Beach FA (1953) Effects of early experience on mating behavior in male rats. J Comp Physiol Psychol 46:204-208.

Kaplan ME, McGinnis MY (1989) Effects of ATD on male sexual behavior and androgen receptor binding: A reexamination of the aromatization hupothesis. Horm Behav 23:10-26.

Kirk RE (1968) Experimental Design: Procedures for the Behavioral Sciences. Brooks/Cole, Belmont, California.

Larsson K (1956) Conditioning and Sexual Behavior in the Male Albino Rat. Acta Psychologia Gothoburgensia, Vol 1. Almqvist \& Wiksell, Stockholm.

Lieberburg I, Wallach G, McEwen BS (1977) The effects of an inhibitor of aromatization (1,4,6androstatriene-3,17-dione) and an anti-estrogen (CI 628) on in vivo formed testosterone metabolites recovered from neonatal brain tissues and purified cell nuclei. Implications for sexual differentiation of the rat brain. Brain Res 128:176-181.

Slob AK, de Klerk LWL, Brand T (1987) Homosexual and heterosexual partner preference in ovariectomized female rats: Effects of testosterone, estradiol and mating experience. Physiol Behav 41:571-576.

Spevak AM, Quadagno DM, Knoeppel D, Poggio JP (1973) The effects of isolation on sexual and social behavior in the rat. Behav Biol 8:63-73.

Tobet SA, Baum MJ (1987) Role of prenatal estrogen in the development of masculine sexual behavior in the male ferret. Horm Behav 21:419-429.

Vega Matuszczyk J (1993) The differentiation of sexual behavior and partner preference of the male rat. PhD thesis, Göteborgs Universitet, Sweden.

Vreeburg JTM, van der Vaart PDM, van der Schoot P (1977) Prevention of central defeminization but not masculinization in male rats by inhibition neonatally of oestrogen biosynthesis. J Endocrinol 74:375-382.

Ward IL, Reed J (1985) Prenatal stress and prepuberal social rearing conditions interact to determine sexual behavior in male rats. Behav Neurosci 2:301-309.

Zimbardo PG (1958) The effects of early avoidance training and rearing conditions upon the sexual behavior of the male rat. J Comp Physiol Psychol 51:764-769. 